\title{
IT-Didaktik: Selbstorganisiertes Lernen in der IT-Ausbildung und eLearning
}

\author{
Margit Scholl
}

\section{Zusammenfassung}

Die Fähigkeit der Selbstorganisation eines lebensbegleitenden Lernens wird immer entscheidender für die Mitarbeiter von Unternehmen und modernen Verwaltungen. Eine Kompetenz, die ebenso im Prozess des eLearnings unabdingbar ist. Daher muss die (Fach-)Hochschulausbildung im IT-Bereich den veränderten Bedingungen angepasst werden, so dass praxisorientiert an aktuellen, komplexen Themen zunehmend selbstorganisiert gelehrt und gelernt werden kann. Mit der Unterrichtsmethode des selbstorganisierten Lernens (SOL) sollte es im Hochschulbereich gezielter als bislang gelingen, Fach-, Methoden- und Sozialkompetenz zur Handlungskompetenz unserer Absolventen zu verschmelzen. Die Rolle der Lehrenden geht uiber in die von Moderatoren, Beratern und Unterstuitzern der individuellen und kooperativen Lernprozesse. Die Qualität der Qualifizierung unserer Absolventen als spätere Mitarbeiter von Wirtschaftsunternehmen oder öffentlicher Verwaltungen wird in einer „passgenaueren“ Qualifizierung mit verantwortungsbewusster Fähigkeit zur Selbstorganisation bei beruflichen Herausforderungen münden. Dieser Beitrag fasst den Lehr- und Forschungsgegenstand der Autorin zum Selbstorganisierten Lernen unter grundsätzlichen Aspekten zusammen und bildet somit die „Langfassung“ bisheriger Präsentationen und Vorträge zum Thema. Mit Blick auf eine Integration von SOL und eLearning werden am Ende des Artikels derzeitige Anforderungen an eLearning-Systeme aus der Sicht des selbstorganisierten Lernens zusammengefasst.

\section{Motivation}

Veränderungen in der modernen Berufs- und Arbeitswelt und damit im gesamten Umfeld der Hochschulausbildung geben Anlass, das Lehren und Lernen neu zu überdenken. Das angestrebte selbstorganisierte Lernen (SOL), das im weiteren Verlauf des Beitrags noch eingehend behandelt wird, ist dabei nicht zu verwechseln mit einem gänzlich selbstbestimmten Lernen der Lernenden. Vielmehr wird nach Herold und Landherr [1] angestrebt, den Vorgaben der Lehrenden entsprechend, die Strukturierung des Lernprozesses während der Unterrichtszeit durch die Lernenden selbst in den Mittelpunkt zu stellen. Dieses Konzept der beiden Studiendirektoren wurde von der Autorin in den vergangenen Semestern in ausgewählten Anwendungsfeldern der IT-Ausbildung am Fachbereich Wirtschaft, Verwaltung und Recht (FB WVR) der Technischen Fachhochschule Wildau (TFHW) adaptiert und mit Studierenden der beiden Studiengänge „Verwaltung und
Recht“ (VR) und „Wirtschaft und Recht“ (WR) erprobt, deren Hintergrund die öffentliche Verwaltung und die verwaltungs- bzw. dienstleistungsorientierte Wirtschaft bilden.

Brandenburg war das erste Bundesland der Bundesrepublik Deutschland, das seine ursprünglich interne Ausbildung für den gehobenen nichttechnischen öffentlichen Dienst bereits 1997 externalisierte und als eigenständige, modernisierte Studiengänge an der TFHW etablierte.

Öffentliche Verwaltung und dienstleistungsorientierte Wirtschaft sind die Informationsverarbeiter per se. Deshalb bleibt der Einsatz der Informationstechnik auch in unseren Studiengängen WR und VR weiterhin und vermehrt von großer Bedeutung, selbst wenn einige Studierende die entscheidende Bedeutung der modernen Medien fuir ihr späteres Arbeitsleben anfangs nicht im vollen Umfang erkennen. Aktuelle IT-Aktivitäten werden im Umfeld von WR und VR derzeit unter dem Schlagwort „eGovernment“ bzw. „eGovernance“ gefuihrt, sei es im Zuge der Verwaltungsmodernisierung oder sei es durch diverse aktuelle Online-Projekte von Bund, Ländern und Kommunen, in denen in Zusammenarbeit mit der Wirtschaft beispielsweise Internetportale aufgebaut, Verfahren unter Nutzung der digitalen Signatur getestet und mögliche Abwicklungen von Geschäftsbzw. Verwaltungsprozessen verfolgt werden. Darüber hinaus verweist eine aktuelle eGovernment-Studie des Institute of Electronic Business (IEB) [2] auf einen für unsere Studiengänge interessanten Aspekt beim Aufbau von Portalen der öffentlichen Verwaltung: „Die von den Unternehmen geäußerten Wünsche bezogen sich zu 14,7 \% auf Angebote rund um das Thema Recht. Es sollte ein Informationsportal für Gesetze, Verordnungen und Richtlinien geben, das mit einer intelligenten Suchsoftware das leichte Auffinden von Inhalten mit Agenten ... ermöglicht." Dieser Gegenstandsbereich wird für unsere zukünftigen Verwaltungswirte und Wirtschaftsjuristen somit von nicht zu unterschätzender Bedeutung sein. Die zunehmend praktische Verquickung von Recht und Informatik sollte sich verstärkt in der Ausbildung moderner Studiengänge widerspiegeln, wozu allerdings besondere Rahmenbedingungen notwendig sind.

Veränderungsprozesse der Berufs- und Arbeitswelt sind immer auch mit Lernprozessen verbunden, die zunehmend zum lebensbegleitenden Lernen werden. Alternative Lernwege vor allem auf elektronischem Wege sollen helfen, individuellen Schulungs- und Beratungsbedarf unabhängig vom Ort zeitnah abzudecken und darüber die Qualität der Qualifizierung zu verbessern. Doch der ursprünglichen Euphorie für eLearning folgte die 
Ernüchterung. Denn zum Anforderungskatalog für das eLearning gehören zweifelsfrei auch ausreichende Selbstorganisations- und Medienkompetenz der Lernenden, so dass die Verbindung mit der Frage geschlossen werden kann, welche Erkenntnisse aus dem selbstorganisierten Lernen für eLearning gezogen werden können.

\subsection{Ausgangslage}

Angesichts des Schocks, den das duirftige deutsche Abschneiden in der „Pisa-Studie“ auslöste, greift nicht nur die Bundesregierung nach dem vermeintlich rettenden Strohhalm IT. Auch rund 70 Prozent der deutschen eLearning-Anbieter rechnen mit guten bis sehr guten $\mathrm{Zu}$ kunftsaussichten für das computergestuitzte Lernen [3]. Ein Makel des eLearnings ist dabei nicht nur das Fehlen einer allgemein verbindlichen Definition, was denn nun eLearning eigentlich sein soll, sondern auch der Umstand, dass sich die Diskussionen zu wenig um die Akzeptanz, um Lernerfolge und Methodeneffizienz drehen [4]. Bislang hat man sich auch infolge entsprechender Förderpolitik beim eLearning zu sehr auf das „e“, also auf den Technikeinsatz und die Infrastruktur, konzentriert. Insofern verwundern Ergebnisse weiterer Studien nicht, wonach eLearning bislang nur einen Randbereich der Qualifizierungsmaßnahmen in deutschen Großunternehmen abdeckt und die Lernkultur von den bekannten Verfahren aus Schule, Universität und anderen Bildungseinrichtungen geprägt ist [5], so dass Medien- und Kommunikationstechnologien nur verhalten zum Tragen kommen.

Bleiben wir kurz bei der Lernkultur an Hochschulen. Wie Kollege Geupel treffend beschreibt [6], blieb die Vorlesung als eine von wenigen Veranstaltungen jahrhundertlang unverändert und ist zudem eine für Studierende recht passive Veranstaltung. Kollegen seien oft enttäuscht über die Prüfungsergebnisse und die Studierenden klagten, dass sie viel Gelerntes bald wieder vergessen. Die Industrie vermisse bei den Absolventen Selbstständigkeit, methodisches Vorgehen und Sozialkompetenz. Es stellt sich somit die Frage, wie Wissen langfristig verankert und in Können überfuihrt werden kann?

Verweilen wir kurz bei den Defiziten der Absolventen. Eine Umfrage in 800 Unternehmen [7] ergab aus Sicht deutscher Unternehmer für Schulabsolventen u. a. die folgenden Defizite: Bei 41,4\% wurde das selbstständige Lernen, bei 39,5\% das Verantwortungsbewusstsein und bei $35,9 \%$ das planvolle Arbeiten vermisst. Diese deutlichen Defizite im selbstständigen Lernen und in der Übernahme von Verantwortung sind bemerkenswert - kaum jeder zweite Schulabsolvent wird den Erwartungen der Wirtschaft gerecht. Es stellt sich ebenso die Frage, ob die Schulabsolventen den Erwartungen der Hochschulen bzw. ob unsere Hochschulabsolventen den Erwartungen ihrer zukuinftigen Arbeitgeber entsprechen? DerUnternehmensumfrage zufolge wurden nur 11,7 \% der Schulabsolventen eine mangelnde Teamfähigkeit bescheinigt - hier scheint schulische Gruppenarbeit für den beruflichen Einsatz zu fruchten. Die Autorin vermutet jedoch aus ihren vielfältigen Projektmanagementerfahrungen, dass bei ernsthaftem Hinterfragen dieses Prozentwertes sich herausstellen dürfte, dass hier bestenfalls die Arbeit in Gruppen, wenn nicht gar nur koordinierte Einzelarbeit gemeint ist, denn konkrete Teamfähigkeit hängt auch vom Anspruch ab, entsprechend der in Abb. 1 dargestellten Entwicklungsstufen der Teamentwicklung: Der Fähigkeit zum individuellen Arbeiten folgen die koordinierte Einzelarbeit und die Arbeit in Gruppen; erst danach kann von Arbeit in Teams oder gar von Arbeit in sich selbst steuernden Teams gesprochen werden. SOL stellt mit dem Ziel, sich selbst steuernde Teams zu entwickeln, daher höchste Ansprüche an die Lernenden. Das derzeitig oft praktizierte eLearning kommt dagegen selten über die ersten Stufen der Teamentwicklung hinaus, ist oft gar nur ein eBook statt ein eLearning. Denken wir aber an die Abhängigkeit der Lernfähigkeit vom Alter [8], die besonders hoch ist für Altersgruppen von 20 bis 28 Jahren mit einem Maximum bei ca. 23 Jahren, so ist das in etwa die vorherrschende Altersspanne unserer Studierenden. D. h. gerade die Hochschulen haben hinsichtlich der Lernfähigkeit ihrer Klientel gute Voraussetzungen, Lernerfolge unter Nutzung neuer Medien und moderner Methoden zu erzielen und dauerhaft zu festigen.

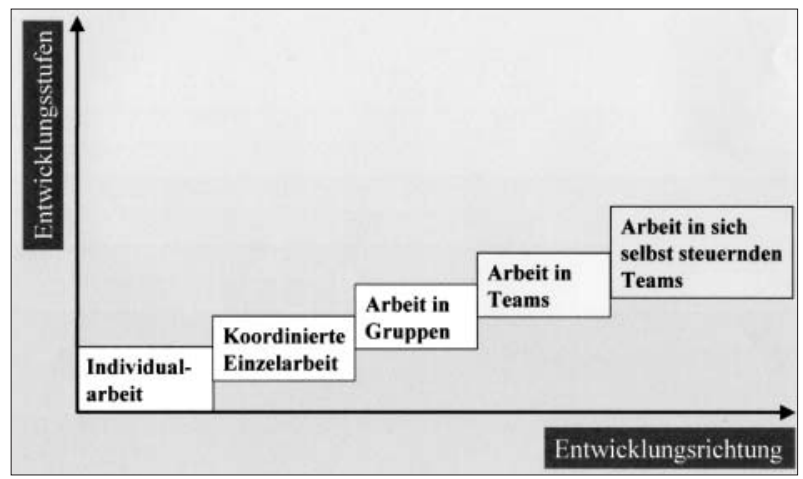

Abb. 1: Teamentwicklung dargestellt nach Diethelm, Projektmanagement, Bd. 1 Grundlagen, Verlag Neue Wirtschafts-Briefe, Herne/Berlin, 2000.

\subsection{Ausbildungsziele}

Aus der skizzierten Ausgangslage kann abgeleitet werden, dass vielen Studierenden das Lernen zum lebensbegleitenden Lernen und die verstärkte Entwicklung der Selbstlernfähigkeit erst vermittelt werden, und dass dazu die Professoren weit mehr als nur ihre fachliche Kompetenz einbringen müssen.

Traditionelle Ausbildungsziele trainieren vorgegebene Arbeitsfolgen sowie Problemlösungsmuster und kontrollierten diese in den Abschlussprüfungen. Nun kommt im Bereich der Informations- und Kommunikationstechnologien das Problem hinzu, dass „Wissen“ bekanntermaßen eine „Halbwertzeit" von ca. drei Jahren hat, d. h. dass Faktenwissen, welches am Anfang der Ausbildung vermittelt wurde, kurz nach der Ausbildung obsolet sein kann. Dieses Problem trifft die in den Berufsalltag drängenden Hochschulabsolventen gleichermaßen wie die einstellenden Unternehmungen, Institutionen oder öffentlichen Verwaltungen. Was müssen Studierende in den unterschiedlichen Studiengängen gegenwärtig überhaupt lernen, um später im innovativen IT-Markt ihrer Branche zu bestehen? So sind es doch beispielsweise die Absolventen unserer Studiengänge WR und VR, die moderne Entwicklungen wie 
„eGovernment“ mit Leben ausfuillen und nutzen, aber auch aus fachlicher Sicht weiter entwickeln sollen. Fähigkeiten und Methodenkenntnisse sind hier vermutlich gleichrangig zu Faktenwissen und Fertigkeiten gefragt.

\subsection{Problemfelder}

Weitere Hintergründe, weshalb man sich mit SOL beschäftigen sollte, bilden allgemein bekannte Untersuchungsergebnisse über die Wechselwirkung zwischen Medieneinsatz und menschlichem Behalten, wonach gemeinsames Sehen und Hören sowie das eigene Tun entscheidende Kriterien für das Lernen und Behalten darstellen. Das, was der Lehrende sagt, wird vom Lernenden weder zwingend gehört noch verstanden. Darüber hinaus hat jeder Mensch seine eigenen Lernpräferenzen und seine bevorzugten Lernkanäle, so dass Lernende gerne in die Lerntypen visueller, auditiver, haptischer und logischer Typ eingeteilt werden [9]. Und je nach Lerntyp wären hinsichtlich notwendiger Qualifizierungsmaßnahmen unterschiedliche Lernwege zu definieren: Ist für den einen ein Seminar zu empfehlen, mag es für den anderen die Nutzung eines Lernprogramms sein. In der Methode SOL wechseln daher unterschiedliche Lernformen in der so genannten „Sandwichstruktur“ miteinander ab und es sollen verschiedenartige Medien zur Stoffvermittlung zum Einsatz kommen.

\subsection{Lernforschung}

Die Ergebnisse der Lernforschung können wie folgt zusammengefasst werden [10]:

- Organisieren Sie den Lernprozess so, dass sich Ihre Lerner viele Erfolgserlebnisse verschaffen können. Schaffen Sie zu Beginn des Lehr-Lernprozesses unterschiedliche positive Motivationen.

- Bringen Sie Ihre Lerner in eine handelnde Auseinandersetzung mit dem Lernstoff.

- Gliedern Sie den Lernstoff in Lernschritte.

- Knüpfen Sie immer an bereits bekannte Informationen an.

- Visualisieren Sie Lernstoffe durch Bilder und Grafiken.

- Sprechen Sie beim Lernen möglichst viele Sinne an!

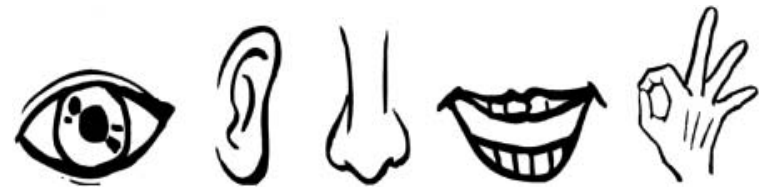

- Im Unterricht sollten kurze Intervalle intensiver Informationsvermittlung mit Übungen und kurzen Pausen wechseln.

Diese Ergebnisse der Lernforschung sind ebenfalls entscheidende Gründe, SOL als Unterrichtsmethode einzufuihren, und sie sollten auch bei der Durchfuihrung von SOL beachtet werden.

\section{Selbstorganisiertes Lernen (SOL)}

Nach Galileo Galilei kann man einem Menschen nichts lehren, man kann ihm nur helfen, es in sich selbst zu entdecken. Auf Grund der in heutiger Zeit üblichen „Massenausbildung“ an unseren Hochschulen, deren bekannte Problemfelder von überfüllten Räumen bis zu erhöhten Abbrecherquoten reichen können, scheint es gerechtfertigt zu sein, dieses ,helfen, es in sich selbst zu entdecken" bewusster als bislang anzugehen, zumal hinsichtlich der Stoffvermittlung die Fragestellungen immer komplexer und interdisziplinärer werden. Mit SOL wird angestrebt, dass die Strukturierung des Lernprozesses während der Unterrichtszeit durch die Lernenden selbst im Mittelpunkt steht.

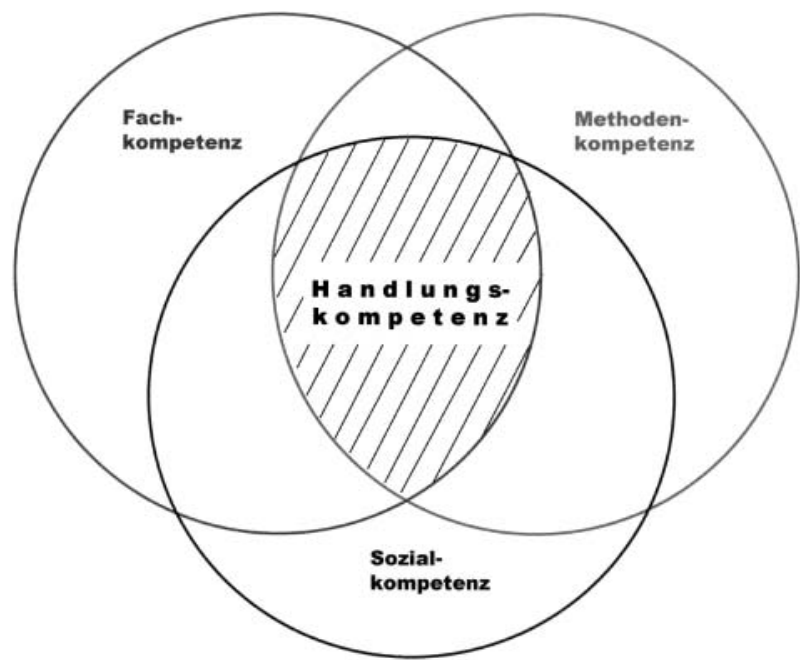

Abb. 2: Entstehen von Handlungskompetenz als Schnittmenge

Wie in der Abb. 2 dargestellt, soll es mit der Unterrichtsmethode selbstorganisierten Lernens auch in der Hochschulausbildung gezielter als bislang gelingen, Fach-, Methoden- und Sozialkompetenz zur Handlungskompetenz unserer Absolventen zu verbinden. Mit SOL sollen Ausbildung und Qualifizierung den Gegebenheiten so angepasst werden, dass die Lernenden mit den spezifischen Anforderungen und Veränderungen der Berufswelt umzugehen lernen und die notwendigen Kenntnisse, Fähigkeiten und Methoden vermittelt bekommen, die sie befähigen, Aufgaben und Probleme ihres späteren beruflichen Handlungsfeldes zielgerichtet, eigenverantwortlich und kompetent zu lösen. Die Zielsetzung von SOL ist somit, verstärkt die Handlungskompetenz der Lernenden (in unserem Fall: der Studierenden) zu entwickeln.

\subsection{Was ist SOL?}

SOL bezieht sich ausschließlich auf den Lernprozess und will neben den fachlichen Inhalten, auch Methoden zur Entwicklung der Selbstlern- und Handlungskompetenz vermitteln. Die Gesamtverantwortung für das Unterrichtsarrangement liegt nach wie vor beim Dozenten, der entsprechend der Döringschen „Kompetenzwanne“ [11], bestehend aus sozialer Kompetenz und Führung sowie organisatorischer, fachlicher, didaktischer und personaler Kompetenz, seine Potenziale in die Vorbereitung und Durchfuihrung einbringt.

Aus Sicht von SOL verliert der Lehrende aber die traditionelle Rolle des Wissensmonopolisten, der linear strukturiertes Wissen auf Vorrat zur Verfuigung stellt - er wird 
Lernberater, der die Lernenden als Lerntypen fördert und unterstiitzt, den kooperativen Lernprozess mit Materialien entsprechend dem gültigen Curriculum vorbereitet und begleitet. Die Lernenden arbeiten abwechselnd individuell und in Teams. Jedes Teammitglied ist für die effektive Arbeit der Gruppe und für die Qualität des Gruppenergebnisses verantwortlich. Spezielle Verantwortungen können in der Gruppe von den Lernenden vereinbart werden. Die Teamleitung beispielsweise moderiert in der gesamten Arbeitsphase, ist für die Arbeitsfähigkeit der Gruppe und für die Dokumentation in Form eines Prozessberichts verantwortlich. Stoffvermittlung, Unterrichtsdurchführung und Lernen werden zum Prozess, der Erkenntnisse der Lernforschung und Teamentwicklung berücksichtigen sollte und die Dozenten nicht aus der Verantwortung lässt.

Nach Herold und Landherr [12] wird das Ideal des selbstbestimmten Lernens im Rahmen der Unterrichtsmethode SOL nicht angestrebt. Vielmehr werden die Lernenden konkret begrenzt, da sie weder die Lerninhalte noch das Lerntempo gänzlich frei bestimmen können und Lehrende nur zu bestimmten Zeiten zur Verfuigung stehen, Lernmittel begrenzt sind, räumliche Engpässe das Bedürfnis nach Ruhe und Konzentration einschränken und klare Vorgaben vom Dozenten als Regeln zu beachten sind. Ein eLearning-System könnte natürlich diese Luicke zwischen selbstorganisiertem und selbstbestimmtem Lernen zu einem gewissen Teil schließen helfen.

\subsection{Wie geht SOL?}

Im Rahmen der Unterrichtsmethode SOL wird nach Herold und Landherr der Lernprozess bewusst als Wechsel zwischen individuellen und kollektiven Lernphasen methodisch geübt und organisatorisch berïicksichtigt. Dieser für ein gutes Lernergebnis erforderliche Wechsel wird als „Sandwicharchitektur" [13] bezeichnet; die eigentliche Wissensvermittlung wird durch das „Gruppenpuzzle“ [14] (engl.: jigsaw) organisiert. Die Vorbereitung fuir diese Form des Lehrens und Lernens, insbesondere unter Berücksichtigung der verschiedenen Lerntypen, unterscheidet sich wesentlich von der einer traditionellen Vorlesung, selbst in ihrer seminaristischen Variante. Da weder Lehrende noch Lernende anfangs mit den erforderlichen Methoden vertraut sind, müssen diese erst sukzessive erworben werden. Es handelt sich somit um einen kontinuierlichen Prozess, sowohl methodisch als auch inhaltlich. Erste Schritte in Richtung SOL erfordern konsequente Hilfestellung in vielerlei Hinsicht:

- klare und uiberschaubare Organisationsstrukturen;

- gute Lernmaterialien und Medien sowie detaillierte Arbeitsaufträge, die Ziele und Inhalte transparent machen;

- weitere Unterstuitzung durch die/den Lehrende(n) hinsichtlich der Methoden für kooperatives Lernen und der Gruppenprozesse.
Lineare Stoffgliederung

5.1 Nervenzellen

Bau, Vorkommen

Potentiale

Erregungsleitung

Bau und Funktion von Synapsen

...

5.2 Lichtsinn

Bau des Wirbeltierauges

Aufbau der Netzhaut

....

5.3 Zentralnervensystem des Menschen Aufbau des Rückenmarks Ablauf eines Reflexes

Gliederung des menschl. Gehirns

5.4 Vegetatives Nervensystem

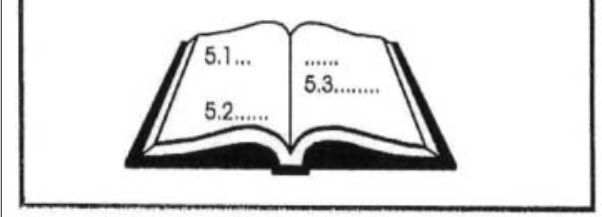

Lehrinhalte werden hintereinander vermillelt

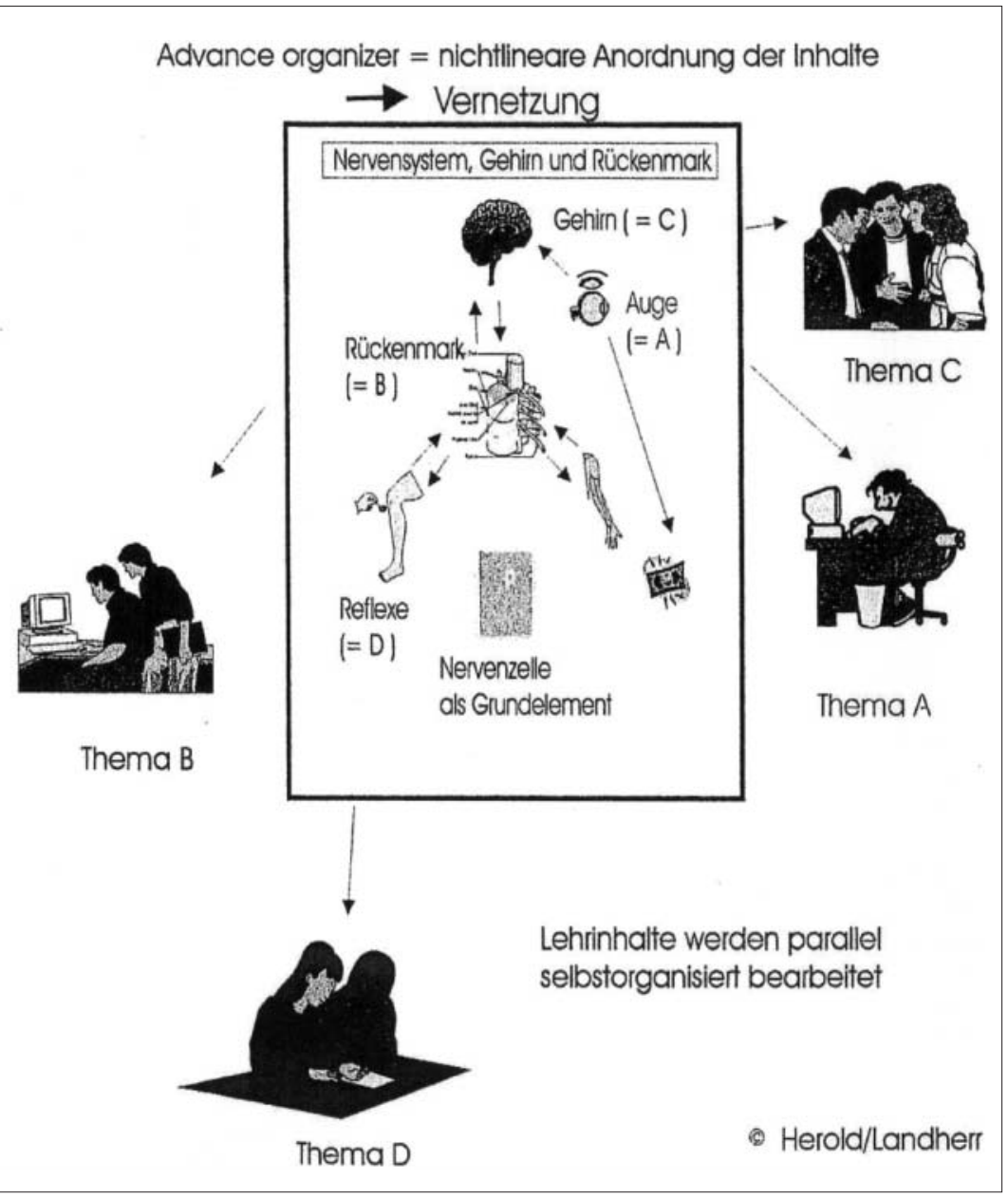

Abb. 3: Advance Organizer (aus Herold und Landherr, a.a.O.) 


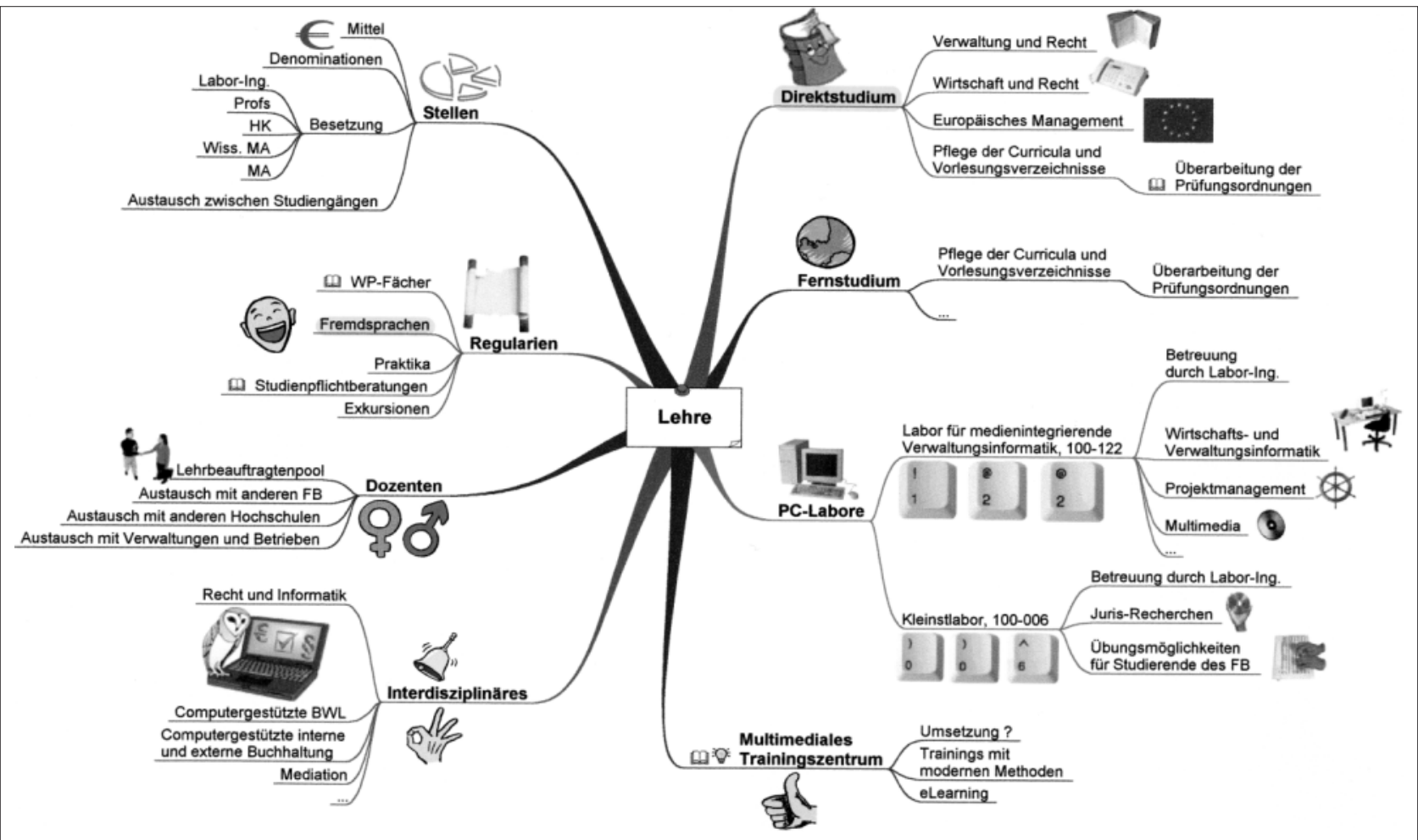

Abb. 4: Aspekte der Lehre am FB WVR als elektronische Mindmap.

\subsubsection{Landkarten im Gehirn und Advanced Organizer (Themenlandkarten)}

Die grundlegende Frage ist, wie Wissen gespeichert wird. Rinke [15] fuihrt aus, dass die beiden Neurochirurgen Penfield und Boldrey bereits im Jahr 1937 unser Gedächtnis als Landkarte beschrieben, auf der all unser Wissen abgebildet ist. Ebenso wie es im Gehirn eine Karte fuir den Körper, also den Tastsinn gibt, existieren modulartig weitere Karten im Gehirn: visuelle Karten zur Abbildung von Bildern, Farben, Bewegung, olfaktorische Karten für den Geruchssinn, gustatorische Karten fuir den Geschmack, akustische Karten für Geräusche, Laute, Frequenzen bis hin zu semantischen Karten, die Bedeutungen abbilden. Umgesetzt in SOL bedeutet es die Entwicklung eines „Advance Organizers“ zu jedem übergreifenden Thema: Statt einer linearen, buchmäßigen Behandlung des Themas (siehe Abb. 3, links) wird eine inhaltliche Lernlandkarte nicht-linear mit Ankerplätzen zu Vorwissen dargestellt (siehe Abb. 3, rechts), die mit Gestaltungselementen unsere Sinne anspricht, Zusammenhänge und Verbindungen darstellt und Assoziationen aufzeigen soll. Auch Döring [16] spricht von der Notwendigkeit der Fixierung gesuchter Ankerbegriffe und ihrer visuellen Darstellung in einer „Fachlandkarte“, aus der Zusammenhänge und Schwerpunkte sofort erkennbar sind. Als Lernen folgt eine ins Einzelne gehende Zerlegung dieser Ankerbegriffe (Detaillierung), die er „Inselbildung“ nennt, und schließlich sei die Bildung von Prototypen für das Lernen entscheidend, zwingende Beispiele also, die die festgelegten Ankerbegriffe enthalten und an denen man den ganzen Sachverhalt beispielhaft erklären kann.

Die Lernlandkarte entsteht praktisch durch Sammlung aller wichtigen Stichworte, Begriffe, Formeln und Daten, die in Cluster strukturiert werden. Sie sollte während der ganzen Lernsequenz den Lernenden präsent und ergänz- bar sein. Wie Herold und Landherr ausfuihren, erleichtern Advance Organizer das Lernen aus folgenden Gründen:

- Die Lernlandkarte fuihrt zu einer gezielteren Aufmerksamkeit, indem sie hilft, die relevanten Informationen auszuwählen;

- sie führt zu einem besseren Verstehen, indem sie den Lernenden in ihrer einzigartigen gedanklichen Struktur hilft, Verbindungen zwischen den neuen Fachthemen und den schon vorhandenen individuellen Wissensstrukturen herzustellen;

- sie hilft den Lernenden, Sachverhalte so aufzufassen, wie sie gemeint sind, und Verwechslungen zu vermeiden, weil sie eine allgemeine gedankliche Struktur bereitstellt.

Darüber hinaus gibt es positive Forschungsergebnisse [17] zu den Wirkungen von Advance Organizer hinsichtlich der Steigerung der Lernleistung in gleicher Lernzeit um 10 bis $18 \%$, ebenso eine Verbesserung des langfristigen Behaltens durch das stabile, kohärente gedankliche Gerüst und einer Steigerung der Transferleistung zu neuen Gebieten um durchschnittlich 10 bis $50 \%$ (je nach Untersuchung). Es bleibt noch zu erwähnen, dass die Reduzierung des Lernstoffs als bekanntes Problem aller Lehrenden durch die Erstellung von solchen Themenlandkarten gelöst werden kann.

Die Autorin regt an, elektronische Mindmaps als Themenlandkarten zu erstellen bzw. zu nutzen, und kann aus Erfahrung auf eine große Akzeptanz verweisen [18]. Diese „eMaps“ basieren auf der Kreativmethode des Mindmappings. Beim Mindmapping als eine bekannte Methode zum visuellen Skizzieren von Gedanken in Vernetzungsform steht im Zentrum das zu behandelnde Thema, dessen zentrale Aspekte in Form von Linien (so genannten Hauptästen), die von diesem Zentrum ausgehen, festgehalten werden. Die zentralen Aspekte werden 
grafisch weiter untergliedert, indem Neben- bzw. Unteräste gezeichnet und entsprechend beschriftet werden, so dass die gesamte Map wie eine von oben betrachtete Baumkrone wirkt (s. Abb. 4 bzw. Abb. 9), wobei die grafischen Elemente angepasst werden können.

Mindmaps werden beginnend bei Zeigerstellung $12 \mathrm{Uhr}$ im Uhrzeigersinn gelesen; innerhalb eines Hauptzweiges linear von oben nach unten. Die Vorteile der elektronischen Map liegen in der für alle Beteiligten sichtbar schnellen Gedankenvisualisierung, damit Förderung der Kreativität, die vor allem über die enthaltene „Drag- und Drop-Funktionalität“ problemlos veränderbar ist. Der Überblick über das gesamte Vorhaben des Brainstormingund Strukuturierungsprozesses bleibt jederzeit erhalten [19]. Die eingebrachten Ideen können schnell und gezielt systematisiert, mit konkreten Zielstellungen textlich untersetzt und mit verschiedenen grafischen Formen kombiniert werden. Auch können die dargestellten Daten medienbruchfrei am PC weiterverarbeitet und in andere Softwareprodukte und Darstellungsformen überführt werden. Als eLearning-Tool kann es eingesetzt werden, indem erklärende Multimediaprodukte mit den Ästen der Mindmap verlinkt werden. Durch weitere Verlinkungen, seien es Dokumente, Webseiten oder E-Mail-Adressen, kann die elektronische Mindmap als Kommunikationszentrum im Intranet oder Internet dienen.

\subsubsection{Gruppenpuzzle (Jigsaw-Gruppen)}

Während die Sandwicharchitektur den für gute Lernergebnisse erforderlichen Wechsel zwischen unterschiedlichen Lernformen sicherstellt, wird die eigentliche Wissensvermittlung durch das „Gruppenpuzzle“ [20] (engl.: jigsaw) organisiert. Die Gruppenpuzzle-Methode wurde seit den 70er Jahren vielfach erprobt und weiterentwickelt. Sie kann sowohl in der Schule als auch in der Erwachsenenbildung eingesetzt werden und ist vor allem dann geeignet, wenn umfangreiche Informationen vermittelt werden sollen. Entscheidend dabei ist, dass sich das zu behandelnde Wissensgebiet in gleichwertige Teilgebiete oder Teilfragen aufteilen lässt, die gemeinsam das Gesamtthema erschließen. Minimum ist die Aufteilung in drei Teile, das Maximum dürfte bei sechs Teilen liegen.

Die Unterrichtsmethode SOL wird von der Autorin, wie bereits erwähnt, am FB WVR seit dem WS 2002/2003 punktuell eingesetzt. Vor allem liegen Erfahrungen im Fach Informations- und Kommunikationssysteme (IKS) vor, das im Studiengang WR im 4. Semester, im Studiengang VR im 6. Semester abgehalten wird und 4 SWS umfasst. Mit SOL wurden hier die Themen „Sicherheitsmanagement“ und „Elektronischer Zahlungsverkehr“" vertieft. Die jeweilige Seminargruppengröße beträgt ca. 40 Teilnehmer/innen. Die Autorin ist allerdings der Ansicht, dass eine themenbezogene Unterrichtsvielfalt entwickelt werden sollte, d. h., SOL mit Gruppenpuzzle stellt nur eine Möglichkeit dar. Konkret wurde in der IT-Ausbildung nicht nur die hier beschriebene Unterrichtsmethode SOL angewendet. Neben Themen, die im seminaristischen Vorlesungsstil behandelt wurden, und klassischen PC-Übungen wurden auch

- Referate mit computergestuitzter Präsentation zum Themenkomplex eGovernment nach dem Online-
Handbuch des Bundesamtes für Sicherheit in der Informationstechnik (BSI) [21] eingesetzt;

- Gruppenarbeiten zum Thema Internet durchgefuihrt, die anhand von zehn Beispielszenarien die Infrastruktur und die Dienste zum Gegenstand hatten;

- Partnergruppen zu vier Fallbeispielen zum Thema Vertragsschluss im Internet eingesetzt, wobei die Lernkontrolle auch über so genannte „Partnerinterviews“ erfolgen kann - hier sollen die Partner im Wechsel pro Frage eine bis zwei Minuten antworten;

- ein Stationenlernen (Lernzirkelprinzip) zum Thema Internetrecherche durchgefuihrt, das unterteilt war in die drei Bereiche Normen/Standards, Datenrecherche (von Suchmaschinen bis Newsgroups) und eCommerce (Shopsysteme und Online-Auktionen). Beim Stationenlernen wird das Themengebiet in Teilbereiche (=Stationen) gegliedert und zu jeder Station werden Informationen zusammengestellt und mehrere Aufgaben formuliert. Jeder Teilnehmer erhält einen „Laufzettel“, auf dem die Stationen verzeichnet sind, die abgearbeitet werden sollen. Einige Stationen müssen als Pflicht bearbeitet werden, andere sind freiwillig. Zum Abschluss wird im Plenum über den Lernfortschritt und die Erkenntnisse diskutiert.

Bei der Gruppenpuzzle-Methode können grob vier Phasen unterschieden werden, die im Folgenden anhand der Abbildungen 5 bis 8 kurz dargestellt und beschrieben werden.

\subsubsection{Phase 1: Einteilung}

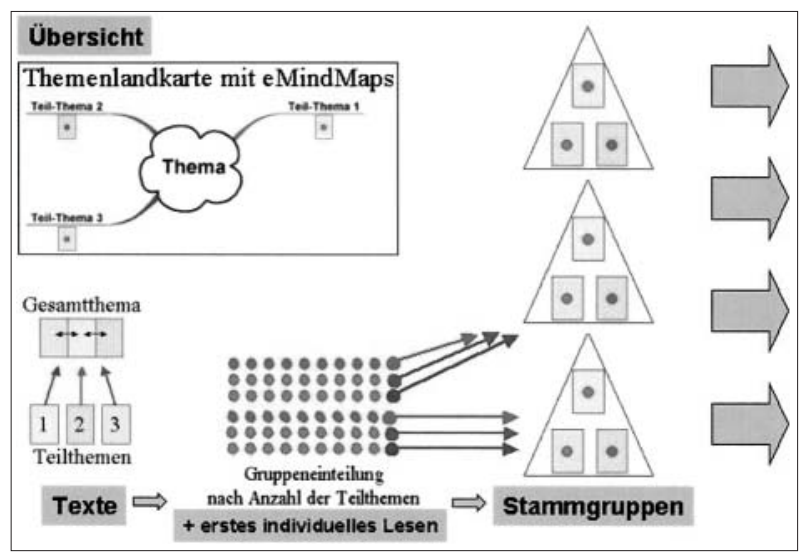

Abb. 5: SOL Phase 1: Schritte zur Stammgruppe

Die Lehrperson (Hochschuldozent) hat das Lernmaterial vorbereitet. Dazu wird der zu vermittelnde Lernstoff (das Wissensgebiet) in mindestens drei (A, B, C) bzw. maximal sechs Teilgebiete zerlegt, die alphabetisch oder nummeriert gekennzeichnet werden. Für jeden dieser Teilbereiche wird Selbststudienmaterial in Form von Texten oder anderen Medien in vergleichbarem Umfang zusammengestellt. Der Unterricht beginnt mit einem Plenum und der Einteilung in Basis- oder Stammgruppen: Die Einteilung kann durch Abzählung oder durch eigene Zuordnung erfolgen. Jedes Gruppenmitglied erhält eine eindeutige Kennung (A, B, C etc.). Jedes Gruppenmitglied erhält nur die Arbeitsmaterialien für das seiner Kennung entsprechende Teilgebiet. Die Lehrperson kann die Verteilung auch mit einem Kurzvortrag beginnen. Der Bezug zum Advance Organizer sollte dargestellt werden, zum 
Beispiel in Form von Mindmap-Übersichten. Nach der Verteilung der Texte bzw. Materialien schließt sich eine kurze individuelle Lernphase (Einzelarbeit, Stillphase) an, in der jeder Teilnehmer seinen Text sichtet.

Zum Gruppenpuzzle mit Expertenlernen wurde konkret das Thema „Sicherheitsmanagement“ von der Autorin anhand eines Lehrbuches in drei Textteile (A, B, C) zu jeweils vier Seiten mit den Schwerpunkten

A: Grundlagen (Grundbedrohungen, Kryptologie, Kryptoanalyse),

B: Chiffiersysteme (symmetrisch, public-key, hybrid),

C: Sicherheitsmaßnahmen (Isolation, Integritätsprüfung, digitale Signatur, digitale Zertifikate, Authentisierung) aufgeteilt. Insgesamt waren für diese erste Phase mindestens 40 Minuten notwendig.

\subsubsection{Phase 2: Expertenlernen}

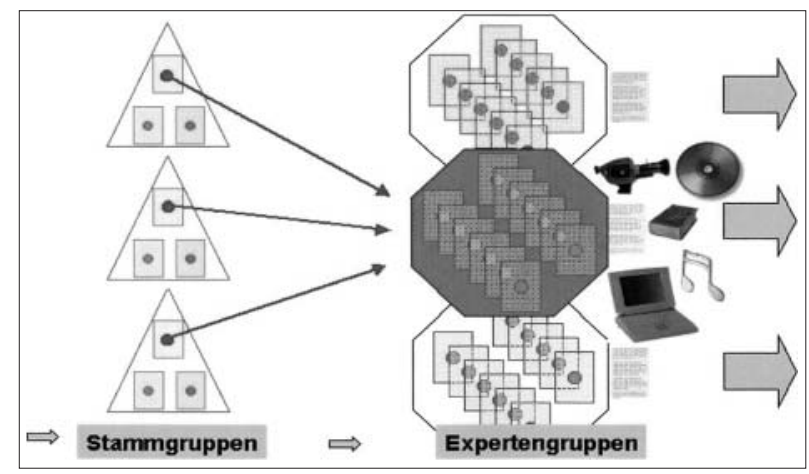

Abb. 6: SOL Phase 2: Schritte zur Expertengruppe

Die eigentliche Wissenserarbeitung wird im anschließenden Expertenlernen organisiert. Dazu diente im Vorfeld die Phase der Einzelarbeit, in der die Studierenden ihr Thema mit dem übergebenen Arbeitsmaterial individuell erarbeiteten. Kleinere Fragen und Tests zur Selbstkontrolle wären möglich und könnten zeigen, ob die Studierenden ihr Thema nach der Individualarbeit schon beherrschen. Es erfolgt nach dieser Phase des Selbststudiums die so genannte Expertenrunde. Mitglieder aus den verschiedenen Stammgruppen, die dasselbe Teilthema bearbeiten, treffen sich in den so genannten Expertengruppen (AAA, $\mathrm{BBB}, \mathrm{CCC})$, um sich für die Vermittlung des Lernstoffes vorzubereiten (s. Abb. 6). Die gemeinsame Erarbeitung des Lernstoffs kann auch durch Recherchen im World Wide Web oder in der Bibliothek ergänzt werden. Ebenso kann die Lehrperson auch ergänzendes Material wie Videos den Expertengruppen zur Verfuigung stellen. Die Expertengruppe kann und soll Infoblätter zu Text, Visualisierungen und/oder Literaturhinweisen als Vermittlungshilfen erstellen. In der „Expertenrunde“ vertiefen und sichern die Studierenden das Gelernte. Sie besprechen also das zuvor individuell Gelernte und beantworten sich gegenseitig noch offene Fragen. Sie unterstuitzen sich somit auf dem Weg zum Experten für das entsprechende Teilgebiet. Über das Fachthema hinaus geht es um die Vermittlung des Gelernten, im Sinne eines „Lernen durch Lehren“. Die Experten bereiten die Vermittlung ihres Teilgebiets (A, B etc.) beispielhaft sprachlich, didaktisch und anschaulich vor. Diese Phase dauerte insgesamt anderthalb bis zwei Stunden. Am Ende kann eine von der Lehrperson vorgegebene Lernkontrolle stattfinden.

\subsubsection{Phase 3: Stammgruppenvermittlung}

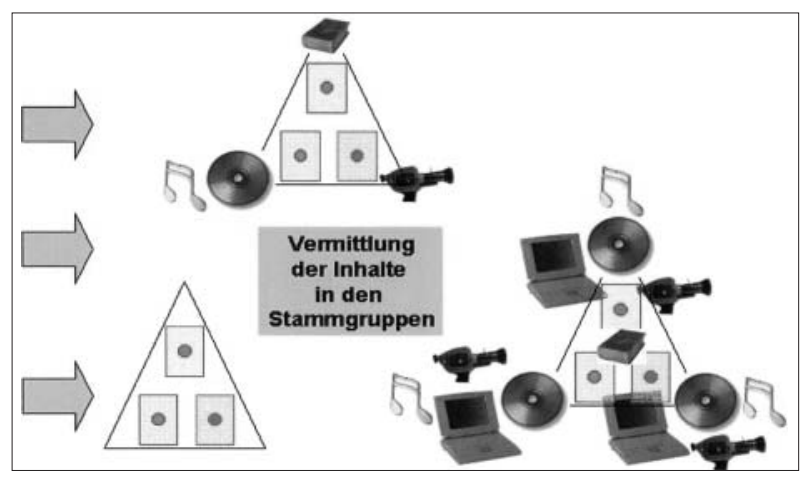

Abb. 7: SOL Phase 3: Stammgruppenvermittlung

In der nachfolgenden dritten Phase geht es um die Vermittlung des Lernstoffs in den Stammgruppen (auch Basisgruppen genannt). In jeder Stammgruppe sind die verschiedenen Teilbereiche des Lernstoffes durch einen Experten vertreten (s. Abb. 7). Reihum unterrichtet jedes Gruppenmitglied als Experte und Lehrperson sein vorbereitetes Thema, während die anderen Gruppenmitglieder jeweils die Lernenden sind. So findet in allen Basisgruppen parallel der gleiche Unterricht statt - bei insgesamt 40 Teilnehmer sollten hierzu größere bzw. mehrere Seminarräume genutzt werden können. Inhaltlich obliegt es jedem einzelnen Stammgruppenmitglied, sein Thema in sinnvoller Art und Weise den anderen Stammgruppenmitgliedern zu vermitteln - dies kann durch unterschiedliche Nutzung vorhandener Materialien durchaus verschieden erfolgen. Insgesamt jedoch haben sich die Studierenden das Thema in strukturierter Weise selbstorganisiert angeeignet. Die Vermittlung des komplexen Lernstoffs „Sicherheitsmanagement“ in den Stammgruppen dauerte maximal eine Stunde; auch hier wurden unterschiedliche Orte genutzt und die Autorin besuchte alle Gruppen reihum.

\subsubsection{Phase 4: Evaluation}

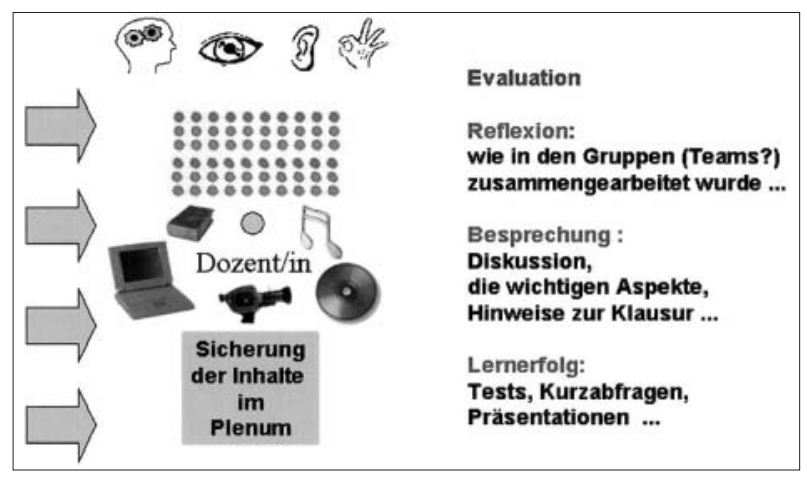

Abb. 8: SOL Phase 4: Plenum

Die in Abb. 8 dargestellte vierte Phase wird von der Lehrperson im Plenum abgehalten. Es erfolgt eine Reflexion, wie in den Gruppen zusammengearbeitet wurde, und eine Diskussion zum Thema, die die wesentlichen Erkenntnisse zusammenfasst. Darüber hinaus standen Flash-Filme zur Verschliusselung und digitalen Signatur zur Verfuigung. Der zusammenfassende Einsatz vielfältiger Medien zum Thema ist sinnvoll, da wir wissen, dass die Lernkurve eines Menschen durch kontinuierliches 
Wiederholen der Vergessensdynamik unseres Gehirns angepasst werden muss: Gelerntes wird zuerst schnell und nach Wiederholung immer langsamer vergessen. Am Ende dieser Phase kann der Lernerfolg durch Tests oder Kurzpräsentationen kontrolliert/evaluiert werden. Es kann ebenso auf eine anstehende Klausur verwiesen werden. Je nach Ausprägung werden eine halbe bis eine Stunde für die Durchfuihrung dieser abschließenden Phase benötigt.

\section{Erkenntnisse}

\subsection{Fazit aus SOL}

Das Ausbildungsziel für die Zukunft muss Handlungskompetenz im neuen, nachhaltigen Sinn sichern. Sie baut auch weiterhin auf Fachkompetenz auf, bezieht aber die fachübergreifenden Qualifikationen der Sozial- und Methodenkompetenz integrierend ein. Der handlungsorientierte Unterricht basiert auf der die Lernenden ins Zentrum stellenden Unterrichtsmethode SOL mit abgestimmten Wechseln von Einzelarbeit, Partnerarbeit, Gruppenarbeit und Arbeit in selbstorganisierten Teams. Für die Unterrichtsmethode SOL ist von Bedeutung, mit kleinen Schritten anzufangen. Die Lehrenden können nicht von Anfang an alle Themen so aufbereitet zur Verfuigung stellen, dass SOL über das gesamte Semester gesehen umfassend praktiziert werden kann. Es ist auch die Frage, ob es dies überhaupt sollte. SOL darf nicht fehlen und muss ausgebaut werden, doch braucht es nicht alles zu ersetzen. Wichtig ist bei der Lehr- und Lernmethode SOL, das Ziel der Selbstständigkeit der Lernenden nicht aus den Augen zu verlieren - hier ist die Selbstständigkeit das Ziel der Unterrichtsmethode, nicht die Voraussetzung! Gleichzeitig liegt eine fraktale Unterrichtsorganisation zu Grunde: Erstens enthält das Organisationsprinzip einfache Grundmuster und eine klare Zielorientierung, Selbstorganisation und Selbstoptimierung sowie eine zugelassene Dynamik. Zweitens werden als didaktische Prinzipien eine Nichtlinearität, Regeln statt direkter Steuerung, Sandwichprinzip, Arbeitsaufträge mit Kompetenzanalyse und Feedback eingeführt. Drittens sind Voraussetzungen sicherzustellen, die Vertrauen schaffen, wie Offenheit aller Maßnahmen und Planungen, klare eigene Ziele, Berechenbarkeit, hohe Akzeptanz der Persönlichkeit der Lernenden. Lernkontrollen sind sowohl zwischen den einzelnen Phasen als auch am Ende möglich.

Die konkrete Erfahrungen aus SOL mit den Lernenden waren folgende:

- Das Prinzip des Gruppenpuzzles wurde schnell verstanden, doch musste die konkrete, verantwortliche Ausfuihrung erst gelernt werden. Etliche Studierende haben die Textfassungen anfangs als zu schwierig empfunden, ein Textverständnis musste erst erworben werden. Hier wird offensichtlich, dass der Einfuihrung ins Thema tatsächlich eine große Bedeutung zukommt und stärker über einen Advance Oganizer, z. B. in Form einer elektronischen Mindmap, erfolgen sollte.
- Begriffe und Abkürzungen wurden zu Beginn nicht eigenständig recherchiert und blieben nicht selten bis zur Klärung durch die beratende Dozentin unklar. Auch konnten die wichtigsten Aspekte der Texte anfangs nicht eigenständig selektiert werden (alles schien gleich wichtig zu sein) und es wurde kaum Methodenvielfalt in der Vermittlung durch die Studierenden sichtbar. Teilweise war während des Expertenlernens eine gewisse Abwehrhaltung gegenuiber dieser Unterrichtsmethode vorhanden, nicht zuletzt deshalb, weil sie als zu anstrengend empfunden wurde.

- Schwierig ist bei dieser Unterrichtsmethode die Raumsituation, im Wintersemester kritischer als im Sommersemester. Ebenso müssen Recherchemöglichkeiten wie Internetzugang und Bibliothek in unmittelbarer Nähe vorhanden sein. Nur vereinzelt wurden eigenständige Materialien zur Vermittlung in den Stammgruppen selbstständig von den Studierenden entwickelt - Lernen durch Lehren muss den Studierenden noch näher gebracht werden, ihre Referatserfahrung reicht dazu nicht aus.

- Alle vier Phasen von SOL müssen an der Hochschule erfolgen, eine Auslagerung der Individualarbeit (Stillphase) nach Hause ist nicht empfehlenswert. Auch die Erfahrungen der FH München zeigen, dass die Ergebnisse eines Alleinlernen der Studierenden mit einem vorgegebenen Lerntext wenig zufriedenstellend waren, weil offensichtlich bei vielen Studenten die Motivation und Selbstdisziplin nicht ausreichten, den Lernstoff außerhalb der Hochschule durchzuarbeiten [22].

- Bei vier SWS erfolgt auf jeden Fall eine Unterbrechung der Lernphase, die zudem sinnvoll gelegt werden muss, während bei Blockunterricht von 6 bis 8 SWS die Themeneinheit komplett abgeschlossen werden kann und hinsichtlich der Gruppendynamik gewisse Vorteile bietet. SOL scheint daher besonders bei Blockunterricht geeignet zu sein.

- Prinzipiell könnte die Unterrichtsmethode SOL jedoch über mehrere Wochen geführt werden, wie es Schulergebnisse zeigen, doch haben Hochschulen kein Anwesenheitszwang, so dass vor allem fuir die Stammgruppen Probleme in der Vermittlungsphase resultieren können, wenn diese beispielsweise nicht mehr anteilig besetzt oder besetzbar sind, so dass Textteile fehlen würden. In solchen Fällen wurden aber problemlos eine neue Aufteilung vorgenommen und Stammgruppen zusammengefasst.

- Als schwierig hat die Autorin die Situation empfunden, wenn Studierende nicht pünktlich zum Unterricht erscheinen, es stört in diesem Falle noch mehr als während einer klassischen Vorlesung.

- Während beim Expertenlernen durchaus Missmut über die hohe Eigeninitiative laut wurde, gab es vor allem in der Stammgruppenvermittlungs- und in der Evaluationsphase viele sehr aktive Teilnehmer/innen mit einem deutlichen Gesamtverständnis für den Inhalt aller Teile, so dass eine überwiegend zufriedene Grundstimmung der Teilnehmer herrschte und das letztendliche Feedback positiv war. Ebenso wurden die entsprechenden Aufgaben in der Klausur überwiegend korrekt beantwortet. Eine statistische Auswertung konnte allerdings nicht erfolgen - dazu 
wäre ein wissenschaftliches (und finanziertes) Projekt mit vergleichenden Gruppen notwendig gewesen. Die Erfahrung deckt sich aber in gewisser Weise mit der aus Heilbronn, wo sich ein bestimmtes selbst gesteuertes Lernen als sehr erfolgreich erwiesen habe [23], das zwar auf Alleinlernen der Studenten beruht, jedoch durch Teamarbeit und ein Teamgespräch mit dem Professor begleitet wird und von den Studenten zur Nachahmung empfohlen wurde, obwohl ihre notwendige Lernaktivität deutlich verstärkt werden musste.

- SOL dauert im Vergleich zur Vorlesung mindestens doppelt so lange, bedarf einer intensiven Vorarbeit und auch hinsichtlich des Prozessablaufs während der Unterrichtszeit ist vor allem bei komplexen Themenstellungen eine intensive Beratung notwendig. Die Aktivierung vieler Studierenden fiel jedoch so positiv auf, dass es für Themenschwerpunkte zu empfehlen ist. Auch dieses Ergebnis entspricht dem aus Heilbronn und der Empfehlung des Kollegen Geupel, in einem Lehrfach drei bis vier Vorlesungen durch ein gesteuertes Lernen zu ersetzen, um den Studenten Gelegenheit zu geben, sich zu einem Team zu formen und Teamfähigkeit zu entwickeln.

Zusammenfassend kann gesagt werden, dass die Unterrichtsmethode SOL dem Anspruch der Lehrenden,

„Problem stellen,

- Literatur/Medien bearbeiten und bewerten,

- Lösungen und Grenzen erarbeiten,

- Ergebnisse frei präsentieren und kritisch würdigen“ eher als andere Vermittlungs- und Übungsformen gerecht wird. Es wird letztlich eine erhöhte Handlungskompetenz unserer Absolventen erreicht, die sich aus Fach-, Sozial- und Methodenkompetenz zusammensetzt. Die Gruppenpuzzle-Methodik erzielt ihre Wirkung nach bisherigen Kenntnissen allerdings nur, wenn der/die Hochschuldozent/in sämtliche Elemente gründlich ausarbeitet und methodisch exakt umsetzt. Diese Methode ist kein entdeckendes Lernen (obwohl dies auch nicht gänzlich ausgeschlossen ist), bei dem die Studierenden ihr Themengebiet selbst finden sollen, sondern dann geeignet, wenn Effektivität in der Informationsvermittlung eine Rolle spielt. SOL als Unterrichtsmethode bedeutet allerdings vermehrte Anstrengung von beiden Seiten, Lehrenden und Lernenden.

\subsection{Fragen zu SOL}

Beide Seiten, die Lernenden und die Lehrenden, müssen sich auf eine solche neue Unterrichtssituation erst einstellen, die vielfach auch verunsichernd wirkt. Sowohl die Vorbereitung als auch die Durchfihrung unterscheiden sich entscheidend von der traditionellen Lehre und bedeuten erst einmal Mehrarbeit für die Dozenten. Die Lehrenden haben eine intensive Vorbereitungsphase mit der Erarbeitung der (Text-)Materialien, die unter inhaltlichen, didaktischen und motivierenden Gesichtpunkten zu entwickeln sind. Vor allem ist es nicht einfach, die Teilgebiete so abzugrenzen, dass einerseits das Gesamtthema umfassend behandelt wird und andererseits die Teilgebietsmaterialien in Gewichtung und Ausfuihrung in etwa gleich sind. In der Durchfuihrungsphase verändert sich mit diesem Konzept die traditionelle Rolle der Lehrenden und sie stehen zur Förderung und Beratung der „Lernteams“ oder auch der Individuen verbindlich zur Verfuigung; diese Hilfestellung müsste aber nicht zwingend genutzt werden. Es stellt sich natürlich die Frage, warum Lehrende diesen Mehraufwand in Kauf nehmen sollten? Nun, eine positive Beantwortung wird wohl durch Motivation und höhere Zielerreichung begründet; von einem veränderten Lehrdeputat wird man wohl nicht ausgehen können.

Die traditionelle Rolle der Lernenden verändert sich weg vom Konsumenten und hin zum aktiv Entscheidenden, der selbst Verantwortung für das eigene Lernen und teilweise auch für das der Mitlernenden übernimmt, selbst organisiert, die Initiative ergreift und eng mit anderen zusammenarbeiten muss. Mit dieser neuen Rolle sind höhere Anforderungen verbunden als beim angeleiteten Lernen in der klassischen frontalen Vorlesung oder im Seminar. Auch hieraus resultiert Mehrarbeit - in diesem Falle für die Studierenden. Wie kann sich der erhöhte Anspruch an aktiv Studierende in der Notengebung wiederfinden? So oder ähnlich fragen die Studierenden, die den hohen Aufwand der SOL-Einheiten und ihre Aktivität belohnt sehen möchten. Gleichzeitig werden unterschiedliche Bewertungsmaßstäbe zwischen Lehrenden und Lernenden über die erbrachte Leistung offensichtlich. Lernende schätzen ihr erhöhtes Engagement oft schon als Erfolgsfaktor ein, der neben dem Klausurergebnis berïcksichtigt werden soll. Und wenn wir die verschiedenen Lerntypen als Faktum ernst nehmen, stellt sich noch eine ganz andere Frage, nämlich, ob fuir alle Studierenden die gleichen Prüfungsaufgaben gestellt werden sollen.

Meist wird im Zusammenhang mit der Diskussion um SOL von Kollegen die Frage gestellt, ob dies denn alles unsere Aufgabe sei. Schließlich seien die Studierenden selbstverantwortlich fuir ihr Studium und wenn ihnen das nicht gelänge, müssten sie die Hochschule eben verlassen. So verständlich diese Argumentation von Kollegen ist, so sehr ignoriert sie zwei entscheidende Aspekte: Zum einen weist Deutschland im internationalen Vergleich von der Anzahl der Studierenden her gesehen deutliche Defizite auf: Wir haben schlicht zu wenig Studierende, um als Wissensgesellschaft und Industrienation nachhaltig bestehen zu können. Und zum anderen legt die klassische Vorlesung die Denkstruktur des Lehrenden als das prägende Element des Lernens zu Grunde. Unterschiedliche Lerntypen haben aber nachweisbar unterschiedliche Denkstrukturen - nur Lernende mit gleichen oder ähnlichen Denkstrukturen wie der Lehrende kommen durch, Lernende mit anderen Denkstrukturen werden als schlecht und Versager definiert. Ist diese Selektion tatsächlich korrekt? Im selbst organisierten Lernprozess gehen mit SOL verschiedene Denkstrukturen ein!

\subsection{Ausblick auf SOL und eLearning}

Themenspezifisch unterschiedlich eingesetzte Unterrichtsmethoden sollen in den nächsten Semestern 


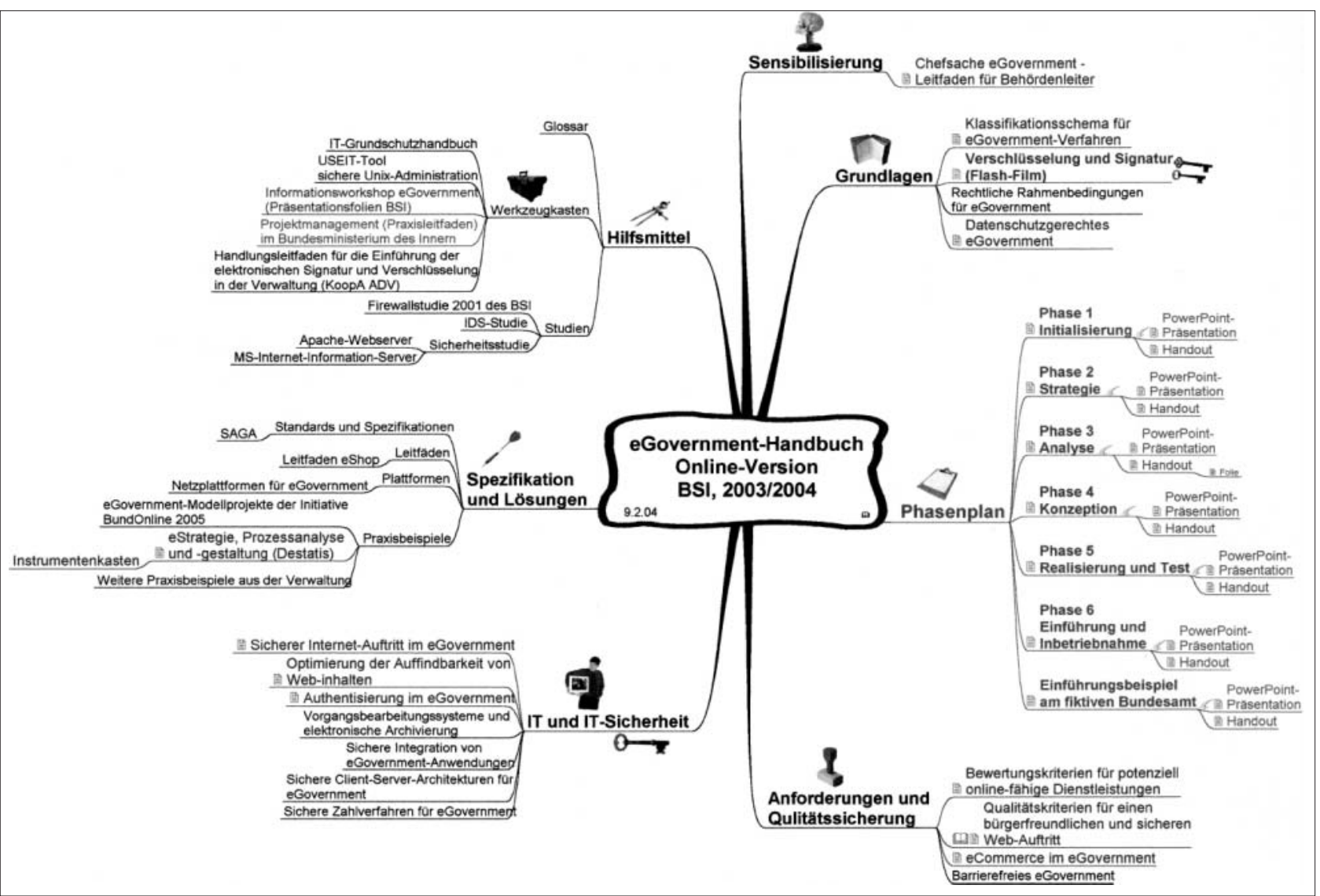

Abb. 9: Darstellung der Gliederung des Online-Handbuchs eGovernment des Bundesamts für Sicherheit in der Informationstechnik (BSI) in Form einer Mindmap und Hinterlegung der einzelnen Äste mit erklärenden Produkten wie Handouts, Tabellen, Flash-Filme, Präsentationen oder Webseiten (siehe auch http://www.tfh-wildau.de/web_wvr/scholl).

weiter unter dem Gesichtspunkt SOL konsolidiert werden. Eine besondere Weiterentwicklung sieht die Autorin in der Nutzung von elektronischen Mindmaps, insbesonders deren Einsatz in der Entwicklung von Advance Organizers für die Einstiegsphasen der zu behandelnden Themen - und zwar unabhängig von der konkret benutzten Unterrichtsmethode. Die praktischen Erfahrungen der Autorin in der Entwicklung und der Nutzung solcher Mindmaps sowohl innerhalb der Hochschule und Lehre als auch im Rahmen von betrieblichen Projektmanagementschulungen und während Beratungen in der öffentlichen Verwaltung zeigen eine allgemein große Akzeptanz bei überaus einfacher Handhabung der benutzten Software, gute Übersichtlichkeit und große Zeitersparnis im Brainstormingprozess. Abbildung 9 zeigt eine solche eMap nicht als spontanes Resultat von Gedankenaustauschen, sondern sie wurde von der Autorin aus der Gliederung des Online-Handbuchs des Bundesamts für Sicherheit in der Informationstechnik (BSI) entwickelt, um das umfangreiche Thema eGovernment in seiner ganzen Komplexität übersichtlicher darzustellen. Diese eMap wurde im Rahmen des Faches Verwaltungsinformatik eingesetzt. Behandelte Themen sind farbig gekennzeichnete Äste, verlinkt mit Materialien wie Handouts, Tabellen, Flash-Filme, Präsentationen oder Webseiten, die von Studierenden erstellt wurden.

Werden die Mindmaps inhaltlich sehr umfangreich, wie in Abbildung 9 zu erkennen, so ist der Aufbau von Multimaps zu empfehlen, die die (lineare) Untergliederung der einzelnen Hauptpunkte in eigenständigen grafischen eMaps zusammenfasst, die selbst über Verlinkung aufrufbar sind. Darüber hinaus könnte für das obige Beispiel auf Basis einer weiterentwickelten „Multimap eGovernment“ eine Verzahnung rechtlicher, betriebswirtschaftlicher und technischer Aspekte dokumentiert werden: Didaktisch aufbereitete Lehrmodule aus verschiedenen Fachdisziplinen, die anfänglich mit Studierenden entwickelt und erprobt wurden, werden in das Thema integriert. Später könnte diese Multimap auch für Weiterbildungen innerhalb der Verwaltungen und Betriebe als CoachingModul genutzt werden (zum Beispiel auch im Rahmen des Netzwerkes NAFöD des Landes Brandenburg, dessen Mitglied die TFHW ist).

Sinnvoll wäre es, wenn die Unterrichtsmethode SOL auch von anderen Dozenten in anderen Fächern sukzessive aufgebaut und praktiziert werden würde. Es würde den Studierenden zunehmend einfacher fallen, selbstorganisiert Verantwortung für den eigenen Lernprozess zu übernehmen, und bliebe nicht eine einmalige Angelegenheit eines bestimmten Faches. In der IT-Ausbildung sollen zur Konsolidierung der bisherigen Erfahrungen auch Schulungserfahrungen aus Videokonferenzen einbezogen werden [24]. In Kooperation mit Kollegen anderer Fachbereiche soll eine an der TFHW entwickelte eLearning-Plattform praktisch unter SOL-Gesichtspunkten genutzt werden. Außerdem werden zurzeit in den PCTrainings elektronische IT-Wissenstests zur Lernkontrolle eingesetzt und es wird geprüft werden, wie sie später in ein Gesamtkonzept zu integrieren sind. 
Aus den bisherigen Erfahrungen mit SOL und unter dem Blickwinkel einer Integration von SOL und eLearning lassen sich derzeit folgende Aspekte für eLearning-Systeme ableiten:

- eLearning sollte über klare Guidelines Hilfestellung geben, damit die richtigen Dinge getan werden können. Ein benutzerfreundliches Glossar unterschiedlicher Schwierigkeitsstufen oder leichte Recherche und Suche sind ebenfalls notwendig.

- Ein verbindlicher, klar strukturierter Rahmen für die Austauschprozesse ist in Verbindung mit den Guidelines zu setzen: eLearning-Tutoren sind notwendig, reichen aber fuir einen nachhaltigen Lernprozess nicht aus.

- Für die Nachhaltigkeit im Lernen ist ein Wechsel von kollektiven und individuellen Lernphasen notwendig: diese Sandwich-Struktur wäre den Lernenden als Lernpfad zu empfehlen und als Guideline im eLearningSystem zu definieren.

- Eine Lernplattform muss somit vielfältige Dienste/ Medien unterstuitzen können und dem Lernenden im Sinne von SOL einen Lernpfad zur Nutzung dieser Dienste anbieten.

- SOL kann gelernt werden, wenn klare und überschaubare Strukturen erkennbar sind. Der Lernstoff muss in Lernschritte gegliedert sein, problemorientiert und personalisiert mit Gesamtuiberblick.

- Der aktuelle Lernstoff sollte jederzeit in einer übergeordneten Themenlandkarte zu erkennen sein; als Visualisierungshilfe kann das elektronische Mindmapping empfohlen werden.

- Es sollte arbeitsbezogene Selektionshilfen geben, denn nicht alles ist gleich wichtig. Eine modulartige Zusammenstellung dessen, was zur Lösung eines Problems notwendig ist, wäre sinnvoll.

- eLearning muss die verschiedenen Lerntypen berüicksichtigen: Der Inhalt (Content) ist von zentraler Bedeutung und für viele Sinne sowie in unterschiedlichen Schwierigkeitsstufen aufzubereiten.

Die zukünftige Aufgabe wird sein, moderne Unterrichtsmethoden wie SOL in eLearning-Systeme zu integrieren. Dieser Entwicklungsprozess aus Theorie und Praxis sowie einer kontinuierlichen Evaluation wird allerdings über Jahre gehen und sollte sich auch bei der Förderung von Forschungsvorhaben umsetzen: Es geht letztlich nicht um Technik, sondern um gute Inhalte und nachhaltige Lernstrategien für unterschiedliche Lerntypen.

\section{Anmerkungen/Literatur}

[1] Martin Herold, Birgit Landherr: Selbstorganisiertes Lernen (Fortbildungsmanuskript). Inzwischen auch als Buch erschienen, Schneider Verlag, 2003, Hohengehren, ISBN 3896766562

[2] Thomas Schildhauer u. a.: E-Government B2G - Anforderungen der Deutschen Wirtschaft, Institute of Electronic Business e. V., Berlin, 2002, S. 57.

[3] Umfrage des High Text Verlags, nach Achim Borns, Ernüchterung, Report E-Learning, iX 5/2002.

[4] Umfrage der D21-Initative, nach Achim Borns, Ernuichterung, Report E-Learning, iX 5/2002.
[5] KPMG-Studie (gemeinsam mit MMB Michel Medienforschung und Psephos), nach Achim Borns Ernuichterung, Report E-Learning, iX 5/2002, S. 120.

[6] Helmut Geupel: Selbst gesteuertes Lernen. Eine - das Lernen aktivierende - Alternative zur Vorlesung, in: hlb die neue Hochschule, 1/2003, S. 16.

[7] Der Tagesspiegel vom 09.12.2001: Was Unternehmen bei Schulabsolventen vermissen, Grafik Tsp/Rita Böttcher.

[8] siehe z. B. Klaus Wolf Döring: Weiterbildung im System. Zur Professionalisierung d. quart. Bildungssektors, Deutscher Studien Verlag, Weinheim 1988.

[9] Transfer GmbH: Lernen mit neuen Medien - ein Lern- und Informationssystem auf CD-ROM, 2001.

[10] Jutta Rothenburg, Peter Müller-Schaefer: Train the Trainer, Trainingsprogramm für Mitarbeiter des Statistischen Landesamtes Berlin, 1994.

[11] Klaus Wolf Döring, Bettina Ritter-Mamczek: Lehren und Trainieren in der Weiterbildung, Deutscher Studien Verlag, Weinheim, 7. Auflage, 1999.

[12] Herold, Landherr, a. a. O.

[13] siehe z. B. Christoph Gerbig, Irene Gerbig-Calcagni: Moderne Didaktik für EDV-Schulungen, Beltz Verlag, Weinheim und Basel, 1998.

[14] z. B. Paul Innerhofer, Sigrid Rotering-Steinberg: Lernen in Schülergruppen, 1988, zit. nach Herold, Landherr, a. a. O.

[15] Michael Rinke: Der IT-Trainer, Addison-Wesley Verlag, München, 2000.

[16] Döring, Ritter-Mamczek, a. a. O.

[17] D. Wahl: Lehrveranstaltungen lerngerecht gestalten, Pädagogische Hochschule Weingarten, 1996.

[18] Margit Scholl, Gerhard Mewes: Kompetenzen und Präsentation des FB WVR - softwaregestuitztes Brainstorming im Fachbereich, TFH-Info 6/2003.

[19] Margit Scholl: eLearning klein, aber fein. Effizienzsteigerung von Brainstormingprozessen in Beratungen durch computergestiitztes MindMapping, Splitter 1/2004; siehe auch http://www.tfh-wildau.de/web_wvr/scholl.

[20] z. B. Innerhofer, Rotering-Steinberg: Lernen in Schülergruppen, 1988, nach Herold, Landherr, a. a. O.

[21] http://www.bsi.de

[22] Geupel, H., a. a. O.

[23] Geupel, H., a. a. O.

[24] Margit Scholl, Sabine Schröter: Customer Support for Job Learning on Demand, in: Wissenschaftliche Beiträge der TFH Wildau, 2004, S. 48 ff.

\section{Autorin}

Prof. Dr. rer. nat. Margit Scholl

Technische Fachhochschule Wildau

Fachbereich Wirtschaft, Verwaltung und Recht

Tel. +493375 508-917

E-Mail: mscholl@wvr.tfh-wildau.de 